\title{
Impactos do estresse na exposição ocupacional de bombeiros: revisão integrativa
}

\author{
Impacts of stress on occupational exposure of firefighters: an integrative review
}

Impactos del estrés en la exposición ocupacional de los bomberos: una revisión integradora

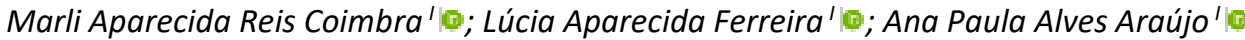

'Universidade Federal do Triângulo Mineiro. Uberaba, MG, Brasil

\begin{abstract}
RESUMO
Objetivo: identificar os impactos na saúde mental de bombeiros relacionados ao estresse da exposição ocupacional. Método: trata-se de uma revisão integrativa da literatura nas bases de dados CINAHL (EBSCO), PsycINFO, MEDLINE ${ }^{\circledR}$, LILACS, Web of Science, Scopus e SCIELO. Para a estratégia de busca utilizou-se os descritores: "Occupational Stress", "Firefighters", "Occupational Exposure". Como critérios de inclusão estabeleceu-se: artigos originais; entre os anos de 2009 e 2019 ; disponíveis completos; estudos transversais; nos idiomas português, inglês e/ou espanhol; e cuja população de estudo incluíssem bombeiros. Resultados: nessa revisão foram analisados 11 artigos. Evidenciou-se que a exposição de bombeiros impactou o desencadeamento de sintomas de depressão e transtorno de estresse pós-traumático. Conclusão: as evidências deste estudo demonstraram que a exposição ocupacional de bombeiros interfere no seu padrão de saúde mental causando estresse por meio do sofrimento psíquico. Este estudo alerta para a necessidade de intervenção e promoção à saúde do bombeiro.
\end{abstract}

Descritores: Bombeiros; Exposição Ocupacional; Estresse Psicológico; Estresse Ocupacional.

\section{ABSTRACT}

Objective: to identify mental health impacts of occupational exposure-related stress among firefighters. Method: this integrative literature review searched the CINAHL (EBSCO), PsycINFO, MEDLINE ${ }^{\circledR}$, LILACS, Web of Science, Scopus and SCIELO databases, suing the descriptors: "Occupational Stress", "Firefighters", "Occupational Exposure". The inclusion criteria were: original articles; between the years 2009 and 2019; full text available; cross-sectional studies; in Portuguese, English and/or Spanish; and whose study population included firefighters. Results: 11 articles were analyzed. Firefighters' exposure was found to impact the triggering of depressive symptoms and post-traumatic stress disorder. Conclusion: the evidence in this study demonstrated that firefighters' occupational exposure interferes with their mental health patterns, causing stress through psychological suffering. This study warns of the need for intervention and promotion of firefighters' health.

Descriptors: Firefighters; Occupational exposure; Psychological Distress; Occupational Stress.

\section{RESUMEN}

Objetivo: identificar los impactos en la salud mental del estrés relacionado con la exposición ocupacional entre los bomberos. Método: esta revisión integradora de la literatura buscó en las bases de datos CINAHL (EBSCO), PsycINFO, MEDLINE ${ }^{\circledR}$, LILACS, Web of Science, Scopus y SCIELO, demandando los descriptores: "Estrés ocupacional", "Bomberos", "Exposición ocupacional". Los criterios de inclusión fueron: artículos originales; entre los años 2009 y 2019; Texto completo disponible; estudios transversales; en portugués, inglés y / o español; y cuya población de estudio incluyó bomberos. Resultados: se analizaron 11 artículos. Se descubrió que la exposición de los bomberos afecta la activación de síntomas depresivos y el trastorno de estrés postraumático. Conclusión: la evidencia de este estudio demostró que la exposición ocupacional de los bomberos interfiere con sus patrones de salud mental, provocando estrés a través del sufrimiento psicológico. Este estudio advierte de la necesidad de intervención y promoción de la salud de los bomberos.

Descriptores: Bomberos; Exposición ocupacional; Distrés Psicológico; Estrés Ocupacional.

\section{INTRODUÇÃO}

Segundo a Organização Mundial de Saúde (OMS) cerca de 300 milhões de pessoas sofrem por depressão, representando $4,4 \%$ da população mundial ${ }^{1}$. $O$ ambiente de trabalho pode desencadear distúrbios físicos e mentais nos trabalhadores, e gerar um custo anual de cerca de 1 trilhão de dólares em perda de produção. Estima-se que para cada 1 dólar investido no tratamento em saúde mental retorne aproximadamente 4 dólares em saúde e produtividade².

A Organização Internacional do Trabalho (OIT) revela que na Europa o estresse ocupacional representa o segundo problema de saúde e afeta cerca de 40 milhões de indivíduos. No Brasil, os transtornos mentais e comportamentais aparecem como a terceira causa de concessão de auxílio-doença e aposentadoria por invalidez ${ }^{3}$.

O estresse ocupacional refere-se às perturbações psicológicas ou sofrimento psíquico pela experiência do trabalho, relacionado às adaptações inadequadas aos eventos estressantes. Também é caracterizado como recorrente e contribui para a incapacidade laboral, além de favorecer a aposentadoria precoce e o risco de suicídio ${ }^{4}$.

Agradecimento pelo fomento concedido pela Pós-Graduação em Atenção à Saúde da UFTM: CAPES - PROAP / Programa de Apoio à Pós-Graduação.

Autora correspondente: Marli Aparecida Reis Coimbra. E-mail: marli.apr.coimbra@gmail.com

Editora responsável: Magda Guimarães de Araújo Faria. 
O sofrimento psíquico, proporcionado pelo estresse no ambiente laboral, surge como uma estratégia de enfrentamento ao não adoecimento e associado à psicodinâmica do trabalho ${ }^{5}$. Trata-se de uma forma de luta do estado emocional e relacionada a sintomas psicológicos e físicos, considerado um transtorno mental comum (TMC), ou seja, não necessariamente ligado a pré-existência de um distúrbio mental. Associa-se a presença de sintomas não psicóticos como insônia, cefaleia, astenia, dor em abdome e sintomas depressivos, que reduzem o bem-estar ocupacional ${ }^{6,7}$.

Neste contexto, percebe-se o bombeiro, uma profissão que lida com situações de estresse no cotidiano de trabalho. Está sujeito ao transtorno de estresse pós-traumático (TEPT) e burnout relacionados às atividades desempenhadas ${ }^{8}$.

Ao corpo de bombeiros compete ser "coordenador e executar as ações de defesa civil, proteção e socorrimento públicos, prevenção e combate a incêndio, perícias de incêndio e explosão em locais de sinistro, busca e salvamento" ${ }^{\prime}$. Além de ter que atender a convocações para atuar em caso de guerra como força terrestre.

Os bombeiros pertencem a um grupo populacional, pouco estudado e exposto a vários eventos estressantes e traumáticos no trabalho. Apresentam taxas altas de TEPT e uso de substâncias como o álcool, em resposta às situações vivenciadas. $O$ uso de álcool e tabaco nesta categoria profissional associa-se a sintomas depressivos ${ }^{10}$.

Estes profissionais estão suscetíveis a exposições tóxicas frequentes de fumaças por combate a incêndios. Respondem a todo tipo de emergência e expostos a riscos ocupacionais de estresse térmico, além de exigência física e mental extenuante, que corrobora para distúrbios mentais ${ }^{11}$. Também os riscos ergonômicos favorecem as lesões por esforço repetitivo, fadiga mental, distúrbios musculoesqueléticos, alterações do sono e má postura dos socorristas ${ }^{12}$. A relação entre trabalho e saúde de bombeiros é abordada em poucos estudos do Brasil, sobretudo em relação à saúde mental destes profissionais ${ }^{13}$.

Assim, este estudo tem relevância social, uma vez que o bombeiro é um agente de segurança pública, responsável por salvaguardar a população e entende-se a importância de fomentar subsídios para civilidade na carreira. Ao considerar os impactos da saúde mental associadas ao trabalho, permitirá aos gestores direcionar ou ressignificar propostas, atitudes e práticas que reduzam o estresse do trabalhador. Em âmbito acadêmico, este trabalho contribui para a compreensão de fatores que interferem na transformação dos processos de trabalho em saúde, educação e segurança. Para tanto, este estudo tem o objetivo de identificar os impactos na saúde mental de bombeiros relacionados ao estresse da exposição ocupacional.

\section{MÉTODO}

Trata-se de uma revisão integrativa da literatura científica, cujo método permite o conhecimento crítico para a prática, fundamentar condutas e orientações para a tomada de decisão ${ }^{14}$.

Para a elaboração seguiu-se as seis etapas propostas para a construção da revisão integrativa ${ }^{14}$ : identificação do tema e elaboração da pergunta norteadora da pesquisa; busca dos artigos na literatura científica e estabelecimento de critérios para inclusão e exclusão de estudos; coleta de dados; análise crítica dos estudos incluídos na revisão; análise e interpretação dos resultados e apresentação da revisão.

A coleta de dados foi realizada no mês de abril de 2020. A seleção dos artigos incluídos na revisão foi realizada por dois revisores de forma independente. Foi definido o tema sobre os impactos na saúde mental de bombeiros relacionados ao estresse da exposição ocupacional. A pergunta norteadora foi desenvolvida pela estratégia PICo ${ }^{15,16}$, que se refere a um acrônimo que significa participante; fenômeno de interesse e contexto. Desse modo considerou-se como "P" os bombeiros, "I" impactos da exposição ocupacional na saúde mental, "Co" ambiente de trabalho estressante. Desta forma a pergunta de pesquisa foi: "Quais são os impactos do estresse da exposição ocupacional sobre a saúde mental de bombeiros?"

Foram estabelecidos como critérios de inclusão os artigos originais publicados entre os anos de 2009 a 2019 desenvolvidos no âmbito nacional e internacional, disponíveis eletronicamente na íntegra e cujos títulos e resumos se referiram à temática do estudo exclusivamente, nos idiomas português, inglês e/ou espanhol, estudos transversais e cuja população de estudo incluíssem bombeiros. Foram excluídos os artigos em duplicata, editoriais, artigos de opinião e de revisão, cartas, comentários, notas, teses, dissertações e manuais ${ }^{14,17}$.

Para garantir a identificação das palavras e o controle do vocabulário, foi realizada consulta aos Descritores em Ciências da Saúde (DeCS), foram selecionados três descritores associados ao tema: "Occupational Stress", "Firefighters", "Occupational Exposure". Após a busca do DECs foi possível pesquisar os descritores no Medical Subject Headings (MESH), PsycINFO Thesaurus e CINAHL Headings.

Para as estratégias de busca fez-se os cruzamentos utilizando-se os descritores selecionados e palavras-chaves correspondentes combinando os métodos booleanos OR e AND de acordo com cada base: ("Occupational Stress" OR 
"Job Stress" OR "Work-related Stress" OR "Workplace Stress" OR "Professional Stress" OR "Job-related Stress") AND ("Firefighters" OR "Fire and Rescue Personnel" OR "Fire Fighters") AND ("Occupational Exposure" OR "Occupational Exposures").

Foram utilizadas as bases de dados a seguir: Literatura Latino-Americana e do Caribe em Ciências da Saúde (LILACS) e Medical Literature Analysis and Retrieval System Online (MEDLINE ${ }^{\circledR}$ ) via Biblioteca Virtual em Saúde (BVS); na Psychology Information - PsycINFO, na base CINAHL with Full Text (EBSCO), Web of Science (Institute for Scientific Information) e Scopus (ambas interface com o portal CAPES); e na base de dados Scientific Electronic Library Online (SCIELO).

Na extração de informações dos artigos, seguiu-se os itens propostos pelo instrumento de Ursi $(2006)^{17}:$ nome do artigo original, características metodológicas, rigor metodológico, intervenções mensuradas e dos resultados ${ }^{14,17}$.

Os artigos incluídos na revisão foram avaliados pelo instrumento padronizado Critical Appraisal Skills Programme $(C A S P)^{16}$, que é um checklist que auxilia na análise crítica dos estudos quantitativos, quanto ao rigor e à credibilidade, segundo os critérios a seguir: objetivo claro; desenho metodológico apropriado aos objetivos; procedimentos metodológicos; seleção adequada da amostra; coleta de dados descrita; relação entre pesquisador e pesquisado; aspectos éticos; análise dos dados fundamentada; resultados apresentados e discutidos; relevância da pesquisa.

Foi avaliada a força e o tipo de evidência dos artigos ${ }^{16}$. Para a análise e posterior síntese dos artigos que atenderam aos critérios de inclusão e para formação de quadro sinóptico foi utilizado: país e ano; método do estudo/participantes; resultados/conclusões ${ }^{17}$. Nesta etapa visou reunir e sintetizar as evidências disponíveis na literatura, assim como a discussão e conclusão ${ }^{14}$.

\section{RESULTADOS}

Foram encontrados 151 artigos nas bases de dados selecionadas, sendo excluídos 38 artigos duplicados, mantendo apenas uma versão de cada. Após a leitura dos títulos 54 não corresponderam aos critérios de inclusão; quanto à leitura dos resumos 40 foram descartados por não atender a metodologia estabelecida. Portanto, 19 estudos incluídos para leitura na íntegra. Após esta última etapa, 8 não contemplaram os critérios de elegibilidade do estudo, selecionados então 11 artigos nesta revisão integrativa. A quantidade de periódicos selecionados em cada base foi: LILACS$=0, M E D L I N E^{\circledR}=01$, PsycINFO=04, SCIELO=01, CINAHL (EBSCO)= 02, Web of Science= 0 e Scopus=3. Na Figura 1 está representado o diagrama das buscas, como recomendado pelo grupo PRISMA ${ }^{18}$ referente aos estudos selecionados pelos revisores.
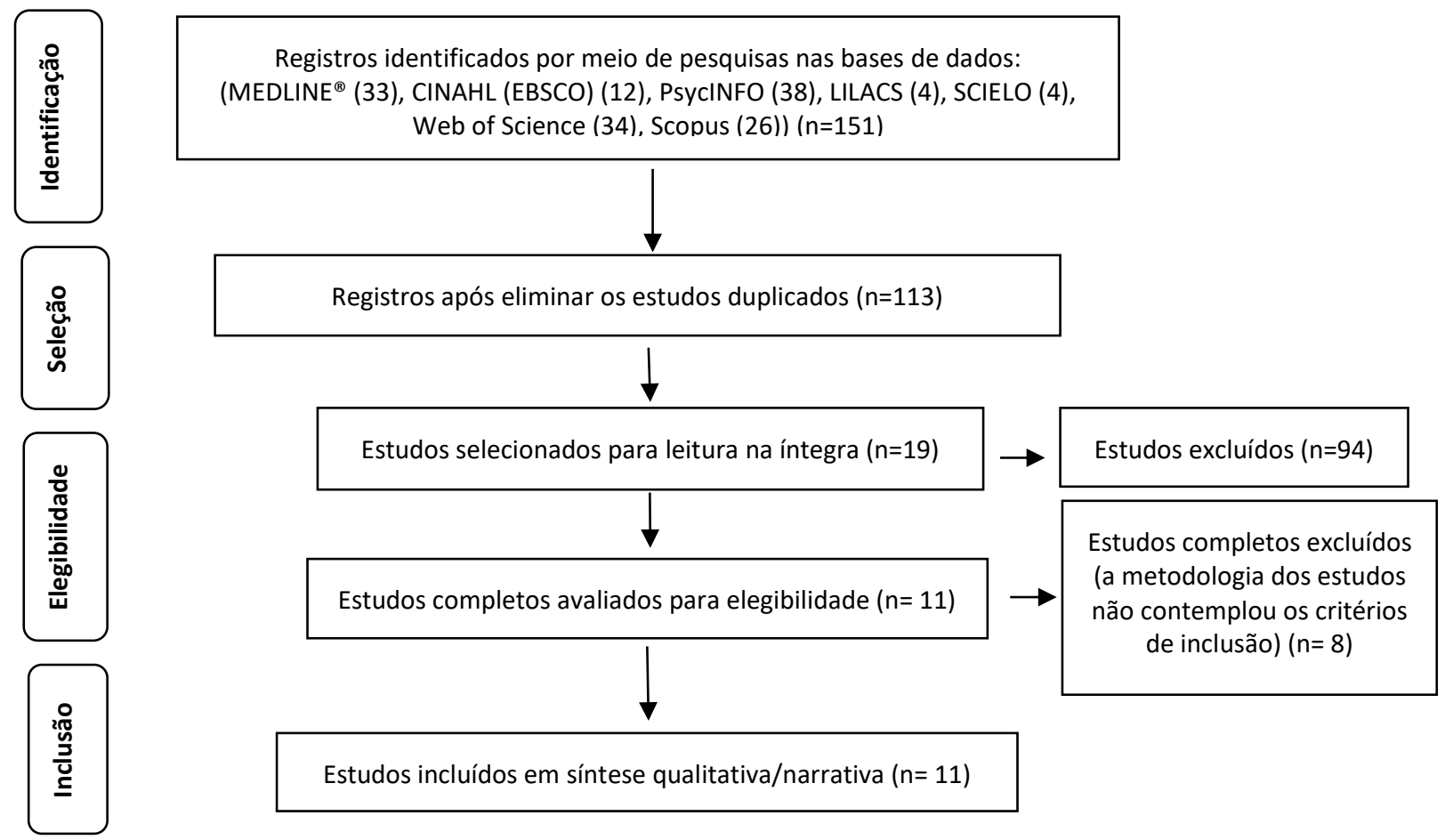

FIGURA 1: Diagrama segundo grupo PRISMA ${ }^{18}$ referente às etapas da seleção dos estudos pelos revisores. 
Os artigos identificados eram de diferentes países (Figura 2), o Brasil e o Canadá corresponderam a mesma proporção de 25\% (2), os demais países (Estados Unidos, Grécia, Jamaica, Guatemala, Alemanha e países europeus) apresentaram a proporção de 12,5\% (1). A Alemanha e a Polônia também estiveram presentes na pesquisa de países europeus. Todos os estudos incluídos contemplaram o desenho transversal. Os artigos foram publicados em periódicos distintos incluindo no Brasil o Journal Nurs. UFPE online e Cad. Saúde Pública; dos demais países foram publicados nos periódicos do Women`s Health Issue; Canadian Journal of Behavioral Science; Occupational Medicine; Psychological Trauma: Theory, Research, Practice, and Policy; Journal of Counseling Psychology; Traumatology; Can J Psychiatry; Psychiatr Pol. e Ann Glob Health.

O ano de publicação dos artigos variou de 2014 a 2019 (Figura 2). Quanto à mensuração do tipo e força da evidência de cada estudo incluído na revisão integrativa ${ }^{16}$ foram classificados como nível de evidência 4 (estudos transversais). Nos artigos selecionados foi aplicado o instrumento $C A S P^{16}$ e ambos contemplaram os itens do instrumento e receberam nota acima de 6 pontos, que indica boa qualidade metodológica e redução de viés.

A síntese dos artigos foi agrupada nas Figuras 2 e 3, referentes à caracterização de país, ano, método do estudo/participantes e resultados/conclusões.

\begin{tabular}{|c|c|c|}
\hline País / Ano & Método de estudo / Participantes & Resultados / Conclusões \\
\hline Brasil, $2014^{12}$ & $\begin{array}{l}\text { Estudo transversal. Utilizada lista de } \\
\text { verificação com: idade, sexo, peso e } \\
\text { estatura, jornada de trabalho e } \\
\text { ergonomia, mapeamento dos sintomas } \\
\text { musculoesqueléticos. Realizada com } 60 \\
\text { bombeiros. }\end{array}$ & $\begin{array}{l}56,7 \% \text { dos militares possuíam fadiga mental e } \\
81,7 \% \text { muscular; } 18,3 \% \text { já sofreram algum tipo de } \\
\text { acidente no trabalho. Os riscos ergonômicos estão } \\
\text { relacionados a distúrbios psicológicos, fisiológicos } \\
\text { e do sono, além de lesões musculoesqueléticas. }\end{array}$ \\
\hline Brasil, $2015^{19}$. & $\begin{array}{l}\text { Estudo transversal. Utilizou-se o } \\
\text { Inventário Beck para Depressão (IBD). } \\
\text { Participaram } 711 \text { bombeiros do sexo } \\
\text { masculino. }\end{array}$ & $\begin{array}{l}\text { A prevalência de depressão na amostra estudada } \\
\text { foi } 5,5 \% \text {. E maior nos que relataram sintomas de } \\
\text { estresse pós-traumático e a exposição a situações } \\
\text { extremas no trabalho podem estar associadas à } \\
\text { depressão e uso abusivo de álcool. }\end{array}$ \\
\hline $\begin{array}{l}\text { República Tcheca, } \\
\text { Alemanha, Itália, } \\
\text { Polônia, Espanha, } \\
\text { Suécia, Reino Unido } \\
\text { e Turquia, } 2015^{20} \text {. }\end{array}$ & $\begin{array}{l}\text { Estudo transversal. Utilizou-se o } \\
\text { Behavior, Security, and Culture-First } \\
\text { Responder (BeSeCuFR, o Impact of } \\
\text { Event Scale-Revised (IES-R) e o } \\
\text { Posttraumatic Growth Inventory-Short } \\
\text { Form (PTGI-SF). Participaram } 1916 \\
\text { bombeiros. }\end{array}$ & $\begin{array}{l}\text { Os preditores de sofrimento e crescimento pós- } \\
\text { evento revelaram } 29 \% \text { da variação no estresse pós- } \\
\text { evento e } 26 \% \text { no crescimento. A exposição a } \\
\text { incidentes angustiantes ou traumáticos } \\
\text { relacionados ao trabalho pode resultar em } \\
\text { condições negativas e positivas para os bombeiros. }\end{array}$ \\
\hline Grécia, $2016^{21}$. & $\begin{array}{l}\text { Estudo transversal. Utilizou-se o Self- } \\
\text { Report Questionnaire, o Maslach } \\
\text { Burnout Inventory (MBI) e a versão } \\
\text { Impact of Event Scale-Revised-Greek. } \\
\text { Participaram } 3289 \text { bombeiros. }\end{array}$ & $\begin{array}{l}\text { A idade, a experiência de trabalho e a condição } \\
\text { física estiveram relacionados com a exaustão } \\
\text { psicológica e síndrome de estresse pós-traumático, } \\
\text { mas a condição de estar responsável pela vida das } \\
\text { pessoas esteve relacionado a depressão por } \\
\text { estresse. Eventos traumáticos e as } \\
\text { atividades/obrigações ocupacionais podem } \\
\text { favorecer o desencadeamento de distúrbios } \\
\text { psicológicos como a depressão e problemas } \\
\text { osteomusculares. }\end{array}$ \\
\hline $\begin{array}{l}\text { Estados Unidos, } \\
2017^{11} \text {. }\end{array}$ & $\begin{array}{l}\text { Estudo transversal que utilizou dados } \\
\text { demográficos individuais, questões } \\
\text { sobre uso de álcool, histórico de } \\
\text { ansiedade, lesões ocupacionais, } \\
\text { satisfação no trabalho. Utilizaram } \\
\text { também a Short Depression Scale, o } \\
\text { Trauma Screening Questionnaire. } \\
\text { Participaram } 256 \text { bombeiros. }\end{array}$ & $\begin{array}{l}\text { 17\% dos participantes eram abstinentes de álcool. } \\
\text { Consumir álcool estava fortemente associado a } \\
\text { indicadores de saúde emocional, e } 2,5 \text { vezes mais } \\
\text { chances de diagnosticado por transtorno } \\
\text { depressivo ou sintomas de estresse pós- } \\
\text { traumático. Os altos níveis de estresse ocupacional } \\
\text { e as exposições tóxicas no trabalho de bombeiros } \\
\text { aumentou os distúrbios médicos e de saúde } \\
\text { mental relacionados ao trabalho. }\end{array}$ \\
\hline
\end{tabular}

FIGURA 2: Apresentação da síntese de artigos pulicados entre 2014 e 2017 incluídos na revisão integrativa. Uberaba, MG, Brasil, 2019. 


\begin{tabular}{|c|c|c|}
\hline País / Ano & Método de estudo / Participantes & Resultados / Conclusões \\
\hline $\begin{array}{l}\text { Jamaica, } \\
2018^{22} \text {. }\end{array}$ & $\begin{array}{l}\text { Estudo transversal. Utilizou a Job-Specific } \\
\text { Scales Assessment, Exposure Scale } \\
\text { Assessment, Family Contact Scale, Beck } \\
\text { Depression Inventory-II, Posttraumatic Stress } \\
\text { Diagnostic Scale, Self-Blame Subscale, PTCI. } \\
\text { Participaram } 259 \text { profissionais entre } \\
\text { bombeiros, policiais e examinadores médicos. }\end{array}$ & $\begin{array}{l}\text { Os resultados apresentaram aumento de cognições negativas } \\
\text { relacionadas à exposição ao trauma no local de trabalho com } \\
\text { sintomas de depressão transtorno de estresse pós-traumático. } \\
\text { A exposição a traumas no local de trabalho tem efeitos nos } \\
\text { processos sociocognitivos que contribuem para os riscos à } \\
\text { saúde mental, como a depressão e transtorno de estresse pós- } \\
\text { traumático. }\end{array}$ \\
\hline $\begin{array}{l}\text { Alemanha, } \\
2018^{23} \text {. }\end{array}$ & $\begin{array}{l}\text { Estudo transversal. Utilizou-se a Self- } \\
\text { Compassion Scale, Patient Health } \\
\text { Questionnaire (PHQ-9-D), Posttraumatic } \\
\text { Diagnostic Scale, validated German version of } \\
\text { the PDS. Participaram } 123 \text { bombeiros. }\end{array}$ & $\begin{array}{l}\text { As contribuições da autocompaixão aos sintomas de } \\
\text { depressão e a eventos estressantes e potencialmente } \\
\text { traumáticos, só surgiu para bombeiros com quantidades } \\
\text { substanciais de experiência no trabalho. Os bombeiros estão } \\
\text { expostos a traumas graves e ao desenvolvimento de sintomas } \\
\text { depressivos, a autocompaixão pode atuar como fator protetor } \\
\text { para aqueles trabalhadores com maior tempo de trabalho. }\end{array}$ \\
\hline $\begin{array}{l}\text { Canadá, } \\
2018^{24} \text {. }\end{array}$ & $\begin{array}{l}\text { Estudo transversal por pesquisa online, } \\
\text { utilizou-se os instrumentos: PTSD (PCL-5), } \\
\text { Depression (PHQ-9), Anxiety (GAD-7), Social } \\
\text { Anxiety Disorder (SIPS), Panic Disorder (PDSS- } \\
\text { SR), Alcohol Use Disorder (AUDIT). } \\
\text { Participaram } 2058 \text { funcionários de segurança } \\
\text { pública (despachantes, trabalhadores } \\
\text { correcionais, bombeiros, policiais e } \\
\text { paramédicos). }\end{array}$ & $\begin{array}{l}\text { Os participantes apresentaram } 44,5 \% \text { de prevalência de } \\
\text { transtorno mental. Este aumento foi associado a maior tempo } \\
\text { de trabalho e também a trabalhadores com mais idade ( }>27 \\
\text { anos). Os que eram mais antigos nos serviços tiveram mais } \\
\text { oportunidades de exposição a } \\
\text { eventos traumáticos. Os bombeiros apresentaram maior } \\
\text { relato de consumo de álcool, associado a mecanismo de } \\
\text { enfrentamento. A prevalência de transtorno mental foi muito } \\
\text { alta em relação a população em geral ( } 10,1 \%) \text {, e sugerem } \\
\text { inclusão de estudo epidemiológico completo, para apoiar a } \\
\text { saúde mental dos trabalhadores de segurança pública. }\end{array}$ \\
\hline $\begin{array}{l}\text { Polônia, } \\
2018^{25} \text {. }\end{array}$ & $\begin{array}{l}\text { Estudo transversal que utilizou: Coping } \\
\text { Inventory for Stressful Situations (CISS) e o } \\
\text { PTSD Interview (PTSD-I) in the Polish language } \\
\text { version (K-PTSD). Participaram } 147 \text { bombeiros. }\end{array}$ & $\begin{array}{l}\text { Quase um terço dos bombeiros da investigação apresentou } \\
\text { sintomas de TEPT, associada a exposição de estresse } \\
\text { traumático durante o serviço. Bombeiros mais velhos ( } 40 \text { a } 49 \\
\text { anos) empreenderam melhor medidas comportamentais para } \\
\text { lidar com a tensão do estresse no trabalho. Recomenda-se } \\
\text { apoio psicológico aos bombeiros mais jovens, com maior } \\
\text { frequência de TEPT. }\end{array}$ \\
\hline $\begin{array}{l}\text { Guatemala, } \\
2018^{26} \text {. }\end{array}$ & $\begin{array}{l}\text { Estudo transversal, responderam questões } \\
\text { sociodemográficas, condições de trabalho e } \\
\text { saúde mental (General Health Questionnaire, } \\
\text { GHQ-12). Participaram } 141 \text { bombeiros. }\end{array}$ & $\begin{array}{l}\text { A exposição à violência no local de trabalho foi de } 37 \% \text {. A } \\
\text { prevalência de sofrimento mental foi maior em bombeiros } \\
\text { expostos à violência ( } 54 \% \text { ). Bombeiros de meia-idade ( } 40-49 \\
\text { anos) apresentaram maior sofrimento por angústia. A } \\
\text { violência está presente no trabalho de bombeiros e associada } \\
\text { a problemas de saúde mental nesta categoria. }\end{array}$ \\
\hline $\begin{array}{l}\text { Canadá, } \\
2019^{27} \text {. }\end{array}$ & $\begin{array}{l}\text { Estudo transversal. Os dados foram coletados } \\
\text { usando uma pesquisa de autorrelato. } \\
\text { Utilizaram também o The Life Events Checklist } \\
\text { for the DSM-5, a PTSD Check List } 5 \text {. } \\
\text { Participaram } 4.441 \text { funcionários de segurança } \\
\text { pública (despachantes, trabalhadores } \\
\text { correcionais, bombeiros, policiais e } \\
\text { paramédicos). }\end{array}$ & $\begin{array}{l}\text { Os participantes relataram exposições a diversos eventos no } \\
\text { trabalho, incluindo morte súbita violenta }(93,8 \%) \text { ou acidental } \\
(93,7 \%) \text {, acidentes graves de transporte }(93,2 \%) \text { e agressões } \\
\text { físicas }(90,6 \%) \text {. A exposição a eventos estressantes no trabalho } \\
\text { esteve associada a diversos transtornos mentais, incluindo, } \\
\text { entre outros, o transtorno do estresse pós-traumático. }\end{array}$ \\
\hline
\end{tabular}

FIGURA 3: Apresentação da síntese de artigos pulicados entre 2018 e 2019 incluídos na revisão integrativa. Uberaba, MG, Brasil, 2019.

\section{DISCUSSÃO}

Nesta revisão foi observado que na avaliação do trabalho de bombeiros, os riscos ergonômicos, como esforços repetitivos no manejo e remoção de pacientes, postura inadequada dos socorristas e plantões com carga horária extensa, contribuíram para distúrbios psicológicos e físicos, além de prejuízo do sono e lesões musculoesqueléticas ${ }^{12}$. As situações de trauma consideradas extremas no ambiente de trabalho destes profissionais associaram-se à sintomas de depressão, de TEPT e uso abusivo de álcool ${ }^{19,24}$. 
Em estudo realizado com funcionários de saúde pública, envolvendo bombeiros, apresentou elevada prevalência de transtorno mental (44,5\%), associada a indivíduos com mais idade e maior tempo de trabalho ${ }^{24}$. Entretanto na Polônia as investigações apontaram que sintomas de TEPT podem ocorrer em profissionais mais jovens e sem experiência em lidar com os traumas do trabalho ${ }^{25}$. Em pesquisa conduzida por 85 estudos realizados em 39 países, para verificar a prevalência de TMC da população mundial, estimou $29,2 \%{ }^{28}$, valor menor em comparação aos agentes de segurança pública.

Pesquisas que identificaram estressores do ambiente ocupacional e sofrimento psíquico de bombeiros evidenciaram que estes eventos experimentados na atividade laboral são fatores de risco para o desenvolvimento de TEPT e sofrimento psicológico ${ }^{26,29-31}$. Quanto ao exercício professional, estão mais suscetíveis a doenças físicas ${ }^{31}$, a tensão por esforço e ainda o trabalho em turnos, com horários de sono irregular, contribuíram para os distúrbios psicológicos e risco de suicídio ${ }^{32}$.

$\mathrm{Na}$ investigação realizada com 1916 bombeiros, de oito países predominantemente europeus, revelou que a exposição aos eventos angustiantes ou traumáticos no trabalho podem gerar adaptações negativas como o desencadeamento de sofrimento psíquico, quanto positivas referentes à capacidade de resiliência do indivíduo ${ }^{20}$. $\mathrm{A}$ própria atividade de rotina da profissão pode contribuir com distúrbios psicológicos como a depressão, além de lesões muscoloesqueléticas ${ }^{21}$.

Segundo a classificação de transtornos mentais e de comportamento da CID-10, vinculada à Organização Mundial da Saúde (OMS), o estresse pós-traumático refere-se às respostas inadequadas ou mal adaptadas ao estresse grave. Trata-se de uma reação ao estresse de eventos realmente ameaçadores (ameaça a segurança de pessoas queridas, catátrofes naturais, acidentes de diversas naturezas, assalto, estupro, mudança súbita de posição social e/ou de relacionamentos interpessoais) ${ }^{33}$.

Em estudo realizado com 4.441 funcionários de segurança pública do Canadá, dos eventos estressantes e geradores de trauma foram incluídos a morte súbita violenta ou acidental, acidentes graves de transporte e agressões físicas $^{24}$. As limitações das demandas de trabalho ou dificuldades em realizar alguma tarefa foi relatada por bombeiros em pesquisa na Coreia com 618 profissionais. Relacionaram-se a eventos traumáticos as ameaças, a lesão pessoal, as fatalidades ou os pacientes feridos. A exposição prolongada e repetida a estes eventos pode comprometer a saúde mental $^{34}$.

A TEPT surge como resposta tardia a uma situação estressante podendo ser de curta ou longa duração, causando angústia nos indivíduos que a experimentam. Os sintomas de TEPT podem incluir desatenção, desorientação, estupor ou agitação e hiperatividade, taquicardia, sudorese, amnésia parcial ou completa, embotamento emocional, afastamento de outras pessoas, ansiedade, depressão, ideação suicida. O uso de álcool ou drogas pode complicar o quadro ${ }^{33}$

Em bombeiros do sexo feminino, foi evidenciado que o uso de álcool esteve relacionado com o estresse e trauma ocupacional ${ }^{11}$. Os indicadores de transtornos de depressão e de TEPT foram associados à exposições tóxicas e eventos traumáticos no trabalho $11,22,27$.

A discriminação e assédio prejudicam a saúde mental e física de mulheres do corpo de bombeiros. Os tipos de assédios sofridos no trabalho são verbais, escritos, sexuais e agressões. As profissionais que sofreram discriminação e assédio moderado a grave apresentaram-se suscetíveis a sintomas depressivos, ansiedade e TEPT e maior problema com o consumo de álcool. Tais associações refletem na insatisfação para o trabalho e ainda no estresse familiar ${ }^{30}$.

Os bombeiros vivenciam o estresse ocupacional e situações tidas como graves que acentuam os sintomas depressivos, porém os que tem mais experiência podem ter ajuda da autocompaixão como estratégia de enfrentamento protetor $^{23}$. Foi observado que para muitos destes profissionais os eventos mais estressantes foram àqueles vivenciados no cotidiano, como incêndios em casas ou acidentes automobilísticos. Situações regulares do trabalho geraram angústias pós-evento estressante e as condições que implicam resiliência e aprendizado após estes fatores depende de condições individuais ${ }^{35}$.

Além do plantão de 24 horas ininterruptos, a rigidez militar, as agressões verbais, os bombeiros ainda enfrentam medo e insegurança da violência urbana, onde as chamadas para atendimento à população podem ser em locais distantes e desconhecidos. Tais situações favorecem o adoecimento e sofrimento destes profissionais ${ }^{13}$.

\section{CONCLUSÃO}

As evidências deste estudo demonstram que a exposição ocupacional de bombeiros gerou o estresse por meio do sofrimento psíquico. Estes profissionais estão expostos a eventos estressantes, traumáticos e ergonômicos no trabalho. As gravidades das ocorrências vivenciadas interferiram no padrão de saúde mental causando danos psicológicos. 
Os principais impactos na saúde mental de bombeiros devido ao estresse da exposição ocupacional foram os sintomas depressivos e a TEPT. Os distúrbios de sono, uso de substâncias como o álcool e lesões osteomusculares também foram relacionados como efeitos da exposição ao trabalho. Contudo, apesar dos eventos traumáticos serem considerados como situações extremas, foi percebido que no trabalho do bombeiro, mesmo em situações de rotina, ocorre a experiência traumática. Foi apontado taxa elevada de transtorno mental e risco de TEPT tanto em profissionais experientes quanto em jovens, portanto a exposição do trabalho contribui para danos à saúde mental.

Embora não tenha sido tema deste estudo, a autocompaixão aparece em um dos estudos como fator protetor de distúrbios mentais em trabalhadores com maior tempo de serviço e, portanto, mais experiências ao trauma. 0 desenvolvimento da resiliência profissional pela exposição ao estresse foi apresentado em um dos artigos, mas dependendo de condições individuais. Entretanto estes dois artigos reforçam a presença de sintomas depressivos pela exposição de traumas no ambiente laboral.

Este estudo alerta para a necessidade de intervenção e promoção à saúde do bombeiro, como forma de amenizar o sofrimento psicológico inerente à profissão. Uma realidade drástica no contexto da sociedade. A importância de gerenciar a organização do trabalho do bombeiro está em prevenir ou amenizar os riscos à saúde mental, e corrobora não apenas com a profissão, mas também com toda segurança pública. Não há pretensão de esgotar o tema, e sim manifestar atenção e cuidado à saúde mental dos bombeiros. Apresenta-se como limitação do estudo o uso de apenas uma base da psicologia e também não foi selecionada nenhuma base da área de administração pública, recomenda-se novos estudos para ampliação da busca e resultados.

\section{REFERÊNCIAS}

1. World Health Organization. Depression and Other Common Mental Disorders: Global Health Estimates. Geneva (Swi): WHO; 2017. [cited $2020 \mathrm{Jul}$ 07]. Available from:

https://www.who.int/mental_health/management/depression/prevalence_global_health_estimates/en/.

2. World Health Organization. Mental health in the workplace. Geneva (Swi): WHO; 2019. [cited 2020 Jul 07]. Available from: https://www.who.int/mental_health/in_the_workplace/en/.

3. Ministério da Saúde (Br). Blog da Saúde. Transtornos mentais são a 3a principal causa de afastamentos de trabalho. Brasília (DF). Ministério da Saúde; 2017. [cited 2020 Jul 07]. Available from:

http://www.blog.saude.gov.br/index.php?option=com_content\&view=article\&id=52979\&catid=579\&ltemid=50218\#: :text=No \%2OBrasil\%2C\%20transtornos\%20mentais\%20e,Previd\%C3\%AAncia\%2FMinist\%C3\%A9rio\%20da\%20Fazenda\%2F2017.

4. Prado CEP. Estresse ocupacional: causas e consequências. Rev. Bras. Med. Trab. [Internet], 2016 [cited 2020 Jul 07]; 14(3):2859. DOI: https://doi.org/10.5327/Z1679-443520163515.

5. Dejours C. Psicodinâmica do trabalho: casos clínicos. Porto Alegre: Dublinense; 2017.

6. Bezerra CM, Assis SG de, Constantino P. Psychological distress and work stress in correctional officers: a literature review. Ciênc Saúde Colet. [Internet], 2016 [cited 2020 Jul 07]; 21 (7): 2135-46. DOI: https://doi.org/10.1590/1413-81232015217.00502016.

7. Graner KM, Cerqueira AT de AR. Integrative review: psychological distress among university students and correlated factors. Ciênc Saúde Colet. [Internet], 2019 [cited 2020 Jul 07]; 24(4):1327-46. DOI: https://doi.org/10.1590/141381232018244.09692017.

8. Santos M, Almeida A. Principais riscos e fatores de risco ocupacionais associados aos bombeiros, eventuais doenças profissionais e medidas de proteção recomendadas. Revista Portuguesa de Saúde Ocupacional. [Internet], 2016 [cited 2020 Jul 07]; 1:43-63. DOI: https://doi.org/10.31252/RPSO.20.01.2016.

9. Corpo de Bombeiros Militar de Minas Gerais (BR). Transição de Governo - Relatórios Setoriais. [Internet]. 2018 [cited 2020 Jul 07]. Available from: http://leisestaduais.com.br/mg/lei-complementar-n-54-1999-minas-gerais-dispoe-sobre-a-organizacaobasica-do-corpo-de-bombeiros-militar-de-minas-gerais-cbmmg-e-da-outras-providencias.

10. Gulliver SB, Zimering R, Knight J, Morissette S, Kamholz B, Meyer E et al. Tobacco and Alcohol Use Among Firefighters During Their First 3 Years of Service. Psychology of Addictive Behaviors. [Internet], 2018 [cited 2020 Jul 07]; 32(3):255-63. DOI: http://dx.doi.org/10.1037/adb0000366.

11. Haddock CK, Poston WSC, Jahnke SA, Jitnarin N. Alcohol use and problem drinking among women firefighters. Women `s Health Issue. [Internet], 2017 [cited 2020 Jul 07]; 27(6):632-8. DOI: http://dx.doi.org/10.1016/j.whi.2017.07.003.

12. Marques CR do CS, Lira M da CC de, Santos Júnior BJ dos, Cruz SL, Lima BRD de A, Lima BRD de A et al. Evaluation of ergonomic risks related to military firefighters' activity. J. Nurs. UFPE on line [Internet], 2014 [cited 2020 Jul 07]; 8(7):3082-9. Available from: https://periodicos.ufpe.br/revistas/revistaenfermagem/article/download/10028/10419.

13. Oliveira MA, Oliveira SS. The hero's human face: analyzing firemen's health-disease process based on virtual communities. Interface (Botucatu) [Internet], 2020 [cited 2020 Jul 07]; 24:e190252. DOI: https://doi.org/10.1590/Interface.190252.

14. Mendes KDS, Silveira RCCP, Galvão CM. Integrative literature review: a research method to incorporate evidence in health care and nursing. Texto Contexto Enferm. [Internet], 2008 [cited 2020 apr 07]; 17(4):758-64. DOI: https://doi.org/10.1590/S010407072008000400018.

15. Santos CMC, Pimenta CAM, Nobre MRC. The pico strategy for the research question construction and evidence search. Rev. Latino-Am. Enfermagem [Internet], 2007 [cited 2019 Jun 14]; 15(3). DOI: https://doi.org/10.1590/S0104-11692007000300023. 
16. Cunha PLP, Cunha CS, Alves PF. Manual de revisão bibliográfica sistemática integrativa: a pesquisa baseada em evidências. [Internet]. Belo Horizonte: Grupo Ănima Educação. 2014 [citado em 07 jul 2020]. Available from: http://biblioteca.cofen.gov.br/wp-content/uploads/2019/06/manual_revisao_bibliografica-sistematica-integrativa.pdf.

17. Ursi ES, Gavão CM. Perioperative prevention of skin injury: an integrative literature review. Rev. Latino-Am. Enferm. [Internet], 2006. [cited 2020 Jul 07]; 14(1):124-31. DOI: https://doi.org/10.1590/S0104-11692006000100017.

18. Moher D, Liberati A, Tetzlaff J, Altman DG. The PRISMA Group. Preferred Reporting Items for Systematic Reviews and MetaAnalyses: The PRISMA Statement. PLoS Med. [Internet], 2009 [cited 2020 Jul 07]; 6(7):e1000097. DOI:. https://doi.org/10.1371/journal.pmed.1000097.

19. Lima EP, Assunção AA, Barreto SM. Prevalence of depression among firefighters. Cad. Saúde Pública [Internet], 2015 [cited 2020 Apr 07]; 31 (4):733-43. DOI: http://dx.doi.org/10.1590/0102-311X00053414.

20. Kehl D, Knuth D, Hulse L, Schmidt S. Predictors of postevent distress and growth among firefighters after work-related emergencies - a cross-national study. Psychological Trauma: Theory, Research, Practice, and Policy. [Internet], 2015 [cited 2020 Apr 07]; 7(3):203-11. DOI: http://dx.doi.org/10.1037/a0037954.

21. Katsavouni F, Bebetsos E, Malliou P, Beneka A. The relationship between burnout, PTSD symptoms and injuries in firefighters. Occupational Medicine. [Internet], 2016. [cited 2020 Apr 07]; 66(1):32-7. DOI: https://doi.org/10.1093/occmed/kqv144.

22. Brondolo E, Eftekharzadeh P, Clifton C, Schwartz JE, Delahanty D. Work-related trauma, alienation, and posttraumatic and depressive symptoms in medical examiner employees. Psychological Trauma: Theory, Research, Practice, and Policy. [Internet], 2018. [cited 2020 Apr 07]; 10 (6): 689-97. DOI: http://dx.doi.org/10.1037/tra0000323.

23. Kaurin A, Schönfelder S, Wessa M. Self-compassion buffers the link between self-criticism and depression in trauma-exposed firefighters. Journal of Counseling Psychology. [Internet], 2018. [cited 2020 apr 07]; 65(4):453-62. DOI: http://dx.doi.org/10.1037/cou0000275.

24. Carleton RN, Afifi TO, Turner S, Taillieu T, Duranceau S, LeBouthillier DM, et al. Mental disorder symptoms among public safety personnel in Canada. Can. J. Psychiatry. [Internet], 2018. [cited 2020 Aug 29]; 63(1):54-64. DOI: http://doi.org/10.1177/0706743717723825.

25. Witt M, Stelcer B, Czarnecka-Iwańczuk M. Stress coping styles in firemen exposed to severe stress. Psychiatr. Pol. [Internet], 2018. [cited 2020 Sep 02]; 52(3):543-55. Doi: https://doi.org/10.12740/PP/73837.

26. Pinto CM, Radon K, van Dijk F. Violence at work and mental distress among firefighters in Guatemala. Ann Glob Health. [Internet], 2018. [cited 2020 Sep 02]; 84(3):532-7. DOI: https://doi.org/10.29024/aogh.2306.

27. Carleton RN, Afifi TO, Taillieu T, Turner S, Krakauer R, Anderson GS et al. Exposures to potentially traumatic events among public safety personnel in Canada. Canadian Journal of Behavioral Science. [Internet], 2019 [cited 2020 Apr 07]; 51(1):37-52. DOI: https://psycnet.apa.org/doi/10.1037/cbs0000115.

28. Steel Z, Marnane C, Iranpour C, Chey T, Jackson JW, Patel V, et al. The global prevalence of common mental disorders: a systematic review and meta-analysis 1980-2013. Int. J. Epidemiol. [Internet], 2014 [cited 2020 Sep 04]; 43(2):476-93. DOI: https://doi.org/10.1093/ije/dyu038.

29. Kyron MJ, Rikkers W, LaMontagne A, Bartlett J, Lawrence D. Work related and nonwork stressors, ptsd, and psychological distress: prevalence and attributable burden among australian police and emergency services employees. Psychological Trauma: Theory, Research, Practice, and Policy. [Internet], 2019 [cited 2020 Jul 07]. DOI: http://dx.doi.org/10.1037/tra0000536.

30. Jahnke AS, Haddock CK, Jitnarin N, Kaipust CM, Hollerbach BS, Poston WSC. The Prevalence and health impacts of frequent work discrimination and harassment among women firefighters in the us fire service. BioMed Research International [Internet], 2019 [cited 2020 Jul 07]; DOI: https://doi.org/10.1155/2019/6740207.

31. Han M, Park S, Park JH, Hwang S, Kim I. Do police officers and firefighters have a higher risk of disease than other public officers? A 13-year nationwide cohort study in South Korea. BMJ Open. [Internet], 2018 [cited 2020 Jul 07]; 8:e019987. DOI: https://doi.org/10.1136/bmjopen-2017-019987.

32. Johnson CC, Vega L, Kohalmi AL, Roth JC, Howell BR, Van Hasselt VB. Enhancing mental health treatment for the firefighter population: understanding fire culture, treatment barriers, practice implications, and research directions. Professional Psychology: Research and Practice. [Internet], 2019 [cited 2020 Jul 07]; 51(3):304-11. DOI: http://dx.doi.org/10.1037/pro0000266.

33. Organização Mundial da Saúde. Classificação de Transtornos Mentais e de comportamento da CID-10: Descrições Clínicas e Diretrizes Diagnósticas. Porto Alegre: Artmed; 2011.

34. Kim M-J, Jeong Y, Choi Y-S, Seo A-R, Ha Y, Seo M, Park K-S. The association of the exposure to work-related traumatic events and work limitations among firefighters: a cross-sectional study. Int. J. Environ. Res. Public Health. [Internet], 2019 [cited 2020 Jul 07]; 16, 756. DOI: https://doi.org/10.3390/ijerph16050756.

35. Kehl D, Knuth D, Holubová M, Hulse L, Schmidt S. Relationships between firefighters' postevent distress and growth at different times after distressing incidents. Traumatology. [Internet], 2014 [cited 2020 Jul 07]; 20(4):253-61. DOI: http://dx.doi.org/10.1037/h0099832. 